\title{
Investigation of ChIP-Seq Technology
} Author: Thomas Yonghee Song Jr. ${ }^{1}$

\author{
E-mail: thomas.songj21@stu.siskorea.org ${ }^{1}$
}

DOI < 10.26821/IJSHRE.8.9.2020.8901>

\begin{abstract}
In the modern context, analysis of the genome is the most efficient for advancements in biology. It has been repeatedly established that nearly every aspect of an individual organism can be traced back to its DNA. The genetic code regulates everything that makes the organism an organism: from expression of phenotypes to embryonic development, all information is stored in the genome. Furthermore, the beauty of the genome is that it can be organized into discrete nitrogenous bases - A, T, G, C for DNA and A, U, G, C for RNA - which creates a viable basis for computational analyses. The development of various bioinformatic technologies is making it possible to create a link between the biological and computational aspects of the genome.
\end{abstract}

Combining Next Generation Sequencing (NGS) and Chromatin Immunoprecipitation (ChIP), collectively labeled ChIP-seq, produces a very powerful tool for mapping protein-binding sites along the DNA. ChIP-seq is capable of providing insight into the mechanisms of gene regulation: the relationship between the genome, proteins, and expressed phenotypes. Furthermore, compared to alternative methods such as ChIP-on-ChIP, ChIPseq is more valuable because of the lack of sequencing-bias and the fact that prior knowledge of the genome is unnecessary; benefits integral to massive parallel sequencing. It can precisely generate millions of counts, making it a popular and cost-effective method.

Together, these two advanced tools produce multiple advantages that are unprecedented in this field of study. Chromatin immunoprecipitation operates by using specific antibodies to precipitate cross-linked DNA fragments and associated protein of interest. As a result, it can isolate specific regions of interest within tens of base pairs of the actual site. In addition, recent developments in NGS allow scientists to accurately sequence millions of DNA fragments on a single run. Thus, when used simultaneously, DNA-binding sites can be mapped easily onto the genome and computational analysis can be executed on the obtained sequences. An important benefit is that ChIP-seq is compatible with the genomes of various organisms in vivo, as well as different forms of data input.

The significance of this bipartite tool is that it pioneers modern research on gene regulation in relation to genetic diseases and biological pathways. With greater accuracy and computational power, the depth of understanding of diseases, such as cancer, and their development increases. Such technology has the potential to produce solutions to diseases of the like that were originally thought to be critical to the human body. By discovering the underlying genetic causes, it becomes easier to develop countermeasures before such phenomena occur.

This paper aims to provide information pertinent to ChIP-Seq and associated technologies, as well as explain their strengths and weaknesses. Rather than an in-depth analyses, we will provide an overview on how ChIP-Seq integrates into modern biomedical research.

Keywords: ChIP-Seq, Chromatin Immunoprecipitation, Next Generation Sequencing, Bioinformatics, Biotechnology, Epigenetics, Histone Modification, DNA, Protein, SISSR's Algorithm 
Volume 8 Issue 9 September 2020

\section{BACKGROUND}

ChIP-seq encompasses chromatin immunoprecipitation and next generation sequencing, both of which have multiple steps of their own.

ChIP-seq is initiated by chromatin immunoprecipitation. This process is done for a specific protein of interest, and it most commonly involves transcription factors or histone modifiers. Specifically, the aim is to determine whether a certain part of the genome is associated with the target protein, possibly mapping out the cistrome. These DNA-protein interactions are vital for "recombination, repair, segregation, chromosomal stability, cell cycle progression, and epigenetic silencing" [1].

The first step of chromatin immunoprecipitation is the fixation of the protein with the chromatin. The fixation of cross-linked DNA and protein is often achieved through formaldehyde, which is optimal due to its efficiency, compatibility with a wide range of protein targets, and reversibility [2]. Fixation by formaldehyde is easily reversible by heat, a characteristic that is exploited in later stages of isolation.

Once the cross-links have been successfully fixated, the chromatin is broken or "sheared" into multiple small pieces, either through sonication or nuclease digestion. It is imperative that the fragments are small (100 500bp) in order to pinpoint the locations of cross-links [1]. Multiple trials across different methods have also demonstrated that longer fixation protocols result in less successful sonication results [3]. In addition, depending on the type of sonicator used, the duration and intensity of sonication needs to be optimized

Once the chromatin has been fragmented, the fragments that contain cross-links need to be identified: this is done through precipitation via antibodies. Also known as immunoprecipitation because it precipitates fragments using antibodies, this is the most important part of the process. Again, it is important to note that the success of ChIP is highly dependent on the type and number of antibodies used [3]. Most of these optimalities are determined empirically.

To complete the process, the fixation is reversed to isolate and purify protein-specific DNA fragments. Because formaldehyde-induced cross-links are easily reversible, incubation at 68 degrees Celsius for 6 hours is enough to separate the proteins [1]; it is the simplest step of ChIP that requires the least optimization. Subsequently, the protein is digested, and the ChIP DNA is purified - residuals are removed through the usage of distilled water. Often, these final fragments are amplified by PCR before being sequenced through NGS. PCR helps to provide a leeway to computational analysis as well as an insight into the level of enriched DNA of a ChIP sample [4]; this is descriptive of the efficiency of the ChIP protocol.

Because ChIP is composed of multiple steps and factors, it is subject to variability. Thus, it is critical that each of these steps are optimized in order to yield optimal results [5]. Rigid control over the variables in each step directly correlates with higher quality in the results. Different types of ChIP also have inherent advantages and disadvantages, which will be discussed later, pertaining to the overall efficacy of ChIP-Seq.

The results of ChIP are compatible with various tools such as different forms of PCR, Southern blot analysis, Western blot analysis, and microarray among others [1]. In ChIP-Seq, they are paired with NGS, which sequences and quantifies the results, thereby facilitating computational analysis.

The capacity of high-throughput sequencing analysis provided by NGS allows users to simultaneously sequence all the ChIP-DNA fragments that have been enriched and purified, as well as to investigate the entire genome for binding sites with high resolution [6]. Though there are numerous different types of NGS, they all operate by sequencing millions of shortened DNA fragments in parallel [7]. Unlike its predecessor, Sanger Sequencing, which can only sequence one fragment at a time, NGS can do millions, making it 
Volume 8 Issue 9 September 2020

a faster, more cost-efficient option for sequencing ChIP-DNA.

While it is one of the most accurate forms of bioinformatics tools on the frontier of biological research, ChIP-seq's processes entail certain inherent biases into its results. Much of this arises from the chromatin structure itself. Since the structural characteristics of chromatin are nonuniform, its fragility varies depending on location. Furthermore, different methods of sonication produce different fragment sizes, resulting in a sample-specific bias. PCR amplification is also largely dependent on the DNA sequence content, as well as the temperature, buffer, and polymerase used. Mapping reads on a reference genome that seems ambiguous or repetitive is also algorithmspecific [8]. These factors all introduce bias to the results of ChIP-seq, and it is necessary to introduce control groups and repetitions to mitigate such biases.

\section{DNA-BINDING PROTEINS}

DNA-binding proteins essentially orchestrate cellular processes because they mediate the biological pathways that occur within and in between cells. Thus, such proteins have a larger implication or organisms, such as humans, in that they dictate the functions of the body. This class mainly encompasses transcription factors (TF) and histone modifications, which are the two items most deeply studied by ChIP-Seq. Problems in TF's can trigger problems or diseases in thousands of genes and the study of histone modifications also provides great insight into epigenomics [9]. By comprehending the sequences and proteins that dictate the body's essential biological pathways, scientists take a step closer to being able to manipulate and emulate these processes.

More specifically, DNA-binding proteins have DNA-binding domains that result in affinity for single or double stranded DNA [10]. Some degree of chromosomal organization can be accomplished without sequence-specific protein interactions. This is largely represented by histones, which form ionic bonds to the backbone of the DNA; the DNA wraps around histones, forming a compact structure known as nucleosome which is the basic unit for chromatin. On the other hand, sequence-specific interactions are often centered around transcription factors, which regulate transcription of DNA to RNA. Transcription factors contain at least one DNA-binding domain and are much more abundant in types: there are about 2000 different transcription factors that regulate genes in the human genome [11].

\subsection{Transcription Factors}

Transcription factors (TFs) are sequence-specific proteins that primarily regulate the processes of gene transcription. Thus, they are invaluable for most biological pathways of organisms. These proteins determine when and how frequently a gene will be used, making them essential for development, cell communication, and protein production. The study of TFs also pioneers research in human stem cells [12].

TFs are trans-acting factors - agents that are not part of the genome itself - that bind to the cis-acting factors - agents that are part of the genome [13]. These proteins are composed of a few domains dedicated to their different functions [14]. As sequence-specific proteins, transcription factors all have a DNA-Binding Domain (DBD) that attaches to enhancers or promoters on the genome. The cis component of this interaction is often called the response element. They also have Trans-Activating Domains (TAD) that bind to other proteins such as coregulators during transcription regulation [15]. Some TFs also have Signal Sensing Domains (SSD) that sense and relay external signals to the transcription complex [16].

The specific domains of the transcription factors help to understand their mechanism. In general, they bind to enhancers or promoters present on the genome to affect the transcription of adjacent genes. Enhancers can be found either upstream or downstream of the gene they regulate as well as up to a million base pairs away from the gene and interacting with multiple different enhancers or genes. In some cases, they affect genes on entirely different chromosomes [17]. Although the specific mechanism of enhancers is still ambiguous, it is 
Volume 8 Issue 9 September 2020

likely that they induce the looping of chromatin to increase transcriptional machinery about the target gene [18]. It is clear, however, that they work together with promoters. Promoters are found upstream of the target gene and are responsible for initiating their transcription [19]. They are composed of a core promoter, proximal promoter, and distal promoter, in increasing distance from the transcription site.

Together with enhancers and promoters, transcription factors induce up- or down-regulation. During up-regulation, the expression of the adjacent gene is increased. Conversely, the transcription of a gene is decreased or completely inhibited during down-regulation. These processes, being essential to the development and maintenance of organisms, are very precise and intricate. Some of the exact properties of regulation mechanisms are not specific. Promoters can be observed acting as enhancers in some cases [20]. Nevertheless, scientists have been able to paint a general picture.

There are multiple specific mechanisms by which gene expression is regulated. Transcription factors often recruit cofactors that activate(coactivator) or repress(corepressor) transcription while binding to the specific enhancer or core promoter regions [21].

General transcription factors (GTF), also known as basal transcription factors, are a class of TFs that are necessary for transcription to occur; they do so by binding to the promoter. These include TFIIA, TFIIB, TFIID, TFIIE, TFIIF, and TFIIH, and are needed to recruit cofactors and facilitate the positioning of RNA Polymerase II along with the core promoter [22]. Usually, TFIID binds to the TATA box in the core promoter, to which TFIIB binds, creating a basis for the transcription complex. TFIIA is sometimes seen to stabilize this interaction. From then on, TFIIE, TFIIF, and TFIIH help complete the complex, the recruited RNA Polymerase II initiates transcription at the transcription start site, and the cofactors mediate the elongation and termination of the transcription [23]. This apparatus is found in sites of transcription upstream of the target gene.
Other TFs regulate this process to control the expression of genes. Often, they do this by affecting the ability of this transcription complex to form. During such forms of regulation, the ability of the GTF to bind with RNA Polymerase II is compromised, resulting in the incomplete formation of the apparatus necessary for transcription [24]. TFs can also catalyze the modification of histones, which controls the availability of a certain gene for transcription (discussed further in the histone modification section). Their binding to enhancers also affects gene expression, because enhancers work with distal promoters to form chromatin looping - increasing the concentration of bodies pertinent to transcription is essential for it to happen [25]. Other than recruiting coactivators and corepressors, TFs also interact through competitive inhibition: the binding of one type of TF to a cisagent prevents the binding of another.

TFs that regulate transcription gene expression are also regulated themselves. Since TFs themselves must also be synthesized through transcription, a negative feedback loop can cause one to repress itself. Binding to its own DNA can induce competitive inhibition, maintaining a certain concentration of the TF. Transcription factors can also be activated or deactivated by direct ligand binding through their SSD or indirect ligand binding on nuclear receptors, as they are often part of signal transduction pathways. Most often, TFs are regulated through the availability of cofactors necessary for their own regulation mechanism, or through the competitive inhibition by different TFs that affect the availability of the DNA-binding site.

Transcription factors are of great functional importance to organisms. Firstly, TFs are essential for cell development and cell differentiation. During the embryonic stage, transcription factors ensure that a stem cell's pluripotency is regulated so that the correct specialization and development occurs [26]. TFs are also responsible for the proper execution of cellular division that is the basis for development and damage repair. Furthermore, they facilitate response to intracellular signals and the environment, as they are often integral to signal transduction or signaling cascades. 
Volume 8 Issue 9 September 2020

Practically, transcriptional regulation largely dictates the conversion from the genotype to the phenotype. Thus, TFs are often regarded with great clinical significance, especially due to the many diseases that are associated with them. Irregular expression of genes is the basis for many different forms of diseases, and one of the most extensively studied ones is cancer. Since TFs regulate the cell cycle, they are often oncogenes or tumor suppressors. In fact, transcriptional regulators account for nearly 20 percent of currently known oncogenes in humans [27]. As a result, scientists are making novel discoveries in the field of cancer previously deemed impossible: targeting the genetic basis of the diseases through TFs provides solutions that simple drugs cannot [28]. Other diseases related to TFs include AIDs, diabetes, and Rett syndrome.

Furthermore, this field of study establishes a strong link to the study of stem cells. Stem cells are pluripotent, as they can practically develop into any type of cell pertinent to an organism. During the embryonic stage, they become specialized through TFs, implying that this process is reversible. In addition, certain TFs are proved to be necessary to maintain this pluripotency, such as POU5F1. This transcription factor is found in the mouse embryo and represses cell differentiation [29]. Previously, scientists would obtain stem cells through isolation and culture of embryonic cells. However, inspection of transcription factors has gradually allowed the selective reprogramming of somatic cells through technologies such as dCAS9. Stem cells have vast therapeutic applications, especially for neurodegenerative diseases such as Alzheimer's and Parkinson's or conditions such as diabetes and leukemia [30]. Scientists can generate stem cells and program them to become a necessary type of specialized cell; subsequently, the cell culture can be transplanted to a patient.

Behind the research between diseases and TFs is ChIP-Seq. Because transcription factors are proteins that bind to DNA, they can be immunoprecipitated through specific antibodies. Efficiently sequencing these sites not only allows scientists to generate a high-resolution, genome wide map of promoters, enhancers, and transcription factors, but also provides insight into their intricate mechanisms.

\subsection{Histone Modifications}

The study of histone modifications is a significant area of study for epigenomics, and it is facilitated through the usage of ChIP-seq. Epigenomic information is not carried in the genome but affects how the DNA template is read and it remains hereditary. In other words, the DNA template is dynamic, and the organization influences how genes are expressed and read. The chromatin is composed of nucleosome units, and these nucleosomes are composed of 2 copies of each core histone - H2A, $\mathrm{H} 2 \mathrm{~B}, \mathrm{H} 3$, and $\mathrm{H} 4$ - wrapped in 1.67 superhelical turns of DNA [31]. Gene expression is controlled mostly by the covalent interactions and modifications of histones [32]. The structure formed through the DNA and histones is called the epigenome, and it is influenced by ancestry as well as environmental factors.

For example, when the DNA is tightly wound around a histone protein, it is harder for transeription to occur, thus repressing the gene expression. If it is lightly wound, transcription occurs more easily, leading to activation of gene expression. As such, while the histone does not directly affect the sequence itself, the histone modifies the sequence's availability to the transcriptional machinery. This is accomplished through post-translational modifications, which include acetylation, methylation, phosphorylation, and ubiquitination [33]. These make significant contributions to organisms' mental and physical attributes.

Each modification operates in different ways. For example, acetylation or deacetylation influences the strength of the electrostatic bond between histone and DNA. This is done through the transfer of acetyl groups - to and from the lysine of the histone - through histone acetyltransferases and histone deacetylases [34]. On the other hand, methylation or demethylation involves the transfer or methyl groups - to and from lysine or arginine residues of the histone - through different methyltransferases. 
Volume 8 Issue 9 September 2020

This results in the indirect recruitment of enzymes that can activate or repress transcriptional activities.

Histone phosphorylation and ubiquitination, in comparison, often work in conjunction with acetylation and/or methylation. During histone phosphorylation, kinases add phosphate groups to histones, and in response, phosphatases remove them. Phosphorylated histones can perform DNA repair, chromatin compaction in mitosis and meiosis, and regulation of transcription [35]. This process also establishes a platform for other histone modifications: for example, histone $\mathrm{H} 3$ phosphorylation influences acetylation levels of the same histone [36]. Histone ubiquitination is executed by DNA ligases by conjugating ubiquitin to histones, which can be removed by deubiquitinating enzymes [37]. Ubiquitination has a critical role in regulating DNA damage signaling, protein translocation, and multiple transcriptional activities such as gene activation and silencing [38]. Like histone phosphorylation, histone ubiquitination also coordinates with acetylation and methylation: for instance, histone $\mathrm{H} 3$ monoubiquitination induces its acetylation [39].

Thus, problematic histone modifications can lead to diseases. Irregular activation or repression of transcription can give rise to tumors and cancers. Specifically, dysregulation of chromatin dynamics through acetylation and methylation is correlated with pathogenesis of cancers, and malfunctions of histone substrates and enzymes through phosphorylation and ubiquitination have been tied with tumorigenesis [40]. For example, the overexpression of MMSET, a type of histone methyltransferase, is associated with a variety of cancers such as multiple myeloma (MM); it is overexpressed in all MM patient samples [41]. MMSET also regulates response to DNA damage [42], and thus interferes with chemotherapy targeted toward MM [40]. Deletion, on the other hand, is found in most patients with - and is a strong candidate for the cause of - Wolf-Hirschhorn syndrome, which is characterized by "mental retardation, craniofacial malformation, and defects in skeletal and heart development" [43]. This example demonstrates the extent of damage that irregularities in a single histone modification can have.

There is an increasing amount of evidence that links epigenomics to diseases such as cancer and AIDS [44]. To this end, ChIP-Seq can be employed to analyze the epigenome. Because ChIP-seq studies the DNA-protein interactions in vivo, it can take a "snapshot" of said interactions. Thus, the chromatin structure formed with histones - the epigenome can be conserved as is, allowing comparisons between different developmental stages, healthy cells, and diseased cells [45]. ChIP-seq will display the locations of DNA-histone interaction, as well as the type of histone modification.

The location and type of histone modification gives insight into the gene and activity associated with it, respectively. Thus, it becomes possible to identify and understand the diseases - such as cancer - that are associated with epigenomics, which is only possible through in vivo analysis. In addition, the comprehension of causes behind such diseases leads to solutions and countermeasures to them.

Epigenomics is closely tied with the lifestyle and environmental factors pertinent to the individual, so ChIP-seq facilitates the formation of not only post-, but also pre- disease development measures.

For example, the study of $\mathrm{H} 3 \mathrm{~K} 4 \mathrm{me} 2$ (dimethylation in 4th lysine residue of histone $\mathrm{H} 3$ ), $\mathrm{H} 3 \mathrm{~K} 4 \mathrm{me} 3$ (trimethylation in 4th lysine residue of histone $\mathrm{H} 3$ ), $\mathrm{H} 3 \mathrm{~K} 9 \mathrm{ac}$ (acetylation in 9th lysine residue of histone $\mathrm{H} 3$ ), and $\mathrm{H} 3 \mathrm{~K} 27 \mathrm{ac}$ (acetylation in 27th lysine residue of histone H3) of Oryza Sativa L. Japonica rice - mainly involved ChIP-Seq. As a result, researchers were able to generate a global map of histone modifications and their positioning along the genome. Compared with a reference genome of rice, it was clear that there was a strong positive correlation between each of these modifications and active transcription or gene expression [46]. Other studies have shown that histone modifications in humans are associated with gene expression of $\mathrm{T}$ cells [47], transcriptomic changes in cancer line cells [48], and pathogenesis in Huntington's Disease [49], among countless more. 
Volume 8 Issue 9 September 2020

\section{SISSRs PEAK-FINDER}

ChIP-Seq alone has its limits to accuracy and bias: the short reads it produces are efficient in filling the genomic map with approximate regions for binding sites, but not the precise sites of binding between DNA protein. As a result, for optimal analysis, there is a need to compare the results from a ChIP-Seq trial to established control data; thus, most mistakes are filtered out. Site Identification from Short Sequence Reads (SISSRs, an algorithm tailored to precise identification of binding sites among the short reads obtained from ChIP-Seq, is tailored for this purpose. It is a highly accurate and specific algorithm that is efficient in filtering out false positives and presenting true binding sites to the user.

As its name implies, SISSRs analyzes the short reads generated from ChIP-Seq protocol and uses them to decide what ones are significant. When the reads are aligned with a reference genome, reads that correspond to unique locations along the genome are retained; those that match with multiple locations are discarded. The remaining reads are commonly called "tags." Tags that are mapped onto the sense strand - the non-template strand - are called "sense tags" whereas tags that are mapped onto the antisense strand - the template strand - are called "antisense tags." Once these tags are established, SISSRs execution preparation is complete.

\subsection{SISSRs Algorithm}

SISSRs is a peak-finder that operates by finding peaks in tags through their density and direction, as well as the average length of sequence strands $(F)$. Using a sliding window of $w$ bases in length, it scans regions containing sequence tags; consecutive windows "slide" or overlap by $w / 2$ bases. For a specific region that is spanned by the sliding window at a given moment, $i$, the net-tag count, $c_{i}$, is computed by subtracting the number of antisense tags from the number of sense tags contained within the window. Candidate binding sites are recorded for $i$ whenever $c_{\mathrm{i}}$ transitions from a positive to negative value. Such sites will be dubbed $t$. These are the namesake peaks identified by the algorithm.
A candidate binding site is retained as a true binding site if and only if specific conditions are met, based on certain parameters that can be manually manipulated.

1. The number of sense tags within $F$ base pairs upstream of $t$ is at least $E$.

2. The number of antisense tags within $F$ base pairs downstream of $t$ is at least $E$.

3. The total number of tags is at least $R$. $R$ is estimated based on a false discovery rate (FDR) that is defined by the user (D) when no control data is available, and an $e$-value threshold if control data is available.

If there is a control dataset, there is a fourth condition that must apply.

4. The ratio of number of tags in the candidate site for the test data to the number of tags in the candidate site for the control đata, also known as the foldenrichment, is at least $p$.

The quality of the control data depends on its saturation. If it does not contain enough reads, then the lack of empirical data taints the statistics used to calculate such threshold values, such as $Z$. On the other hand, if a control dataset is unavailable, the background tag distribution - the distribution of tags outside the candidate site - is modeled through a Poisson distribution [50].

Most of the parameters are predetermined or automatically calculated. The following describes how they are set.

$w$ : The size of the sliding window. Default value is 20. This can be changed by the user.

$E$ : Threshold value for each sense and antisense tags referred to on conditions 1 and 2. Default value is 2 . This can be changed by the user.

$F$ : The average length in base pairs of a precipitated fragment of DNA. This can be input by the user if known or calculated by the algorithm.

$M$ : The mappable genome length. It is estimated to be around 80 percent of the entire genome for humans. 
Volume 8 Issue 9 September 2020

$n$ : Total number of tags in the dataset.

$\lambda$ : The expected number of tags. For a window of length $2 F$-bp, it is calculated as

$2 F \cdot\left(\frac{n}{M}\right)$

$e$ : Euler's number. It is used for expected values, as the Poisson distribution is used to model a background tag distribution based on random chance. It is equal to $\ln 2$ or approximately 2.718 . It should not be confused with the $e$-value threshold.

$e_{t}: e$-value threshold. The threshold value for the false discovery rate used to calculate $R$. It is the expected number of enriched regions for the size of the dataset based on Poisson probabilities. It should not be confused with Euler's number.

Case 1: No control data available

FDR: The false discovery rate. For each integer value $V \geq 2 E$, the $F D R$ is the ratio of the number of $2 F$-bp regions that have at least $V$ tags based on chance (Poisson probability) to the actual number of $2 F$-bp regions that have at least $V$ tags.

$D$ : The threshold value for the false discovery rate used to calculate $R$. It is set by the user.

$R$ : The threshold value for the total number of sense and antisense tags. It is the minimum $V$ (refer to $F D R$ ) such that $F D R<D$.

Case 2: Control data is available

$R$ : The threshold value for the total number of sense and antisense tags. It is the maximum integer value $V \geq 2 E$ such that the number of $2 F$-bp regions that have at least $V$ tags on the control data is less than $e_{t}$.

$p: p$-value threshold. The threshold value for the fold-enrichment value of a candidate site. The value of $p$ is determined by the distribution of foldenrichment values for multiple random sites - at least a million.

Using a Poisson distribution to model the background tag distribution is important to calculating the expected value or probability of tag occurrences and is pertinent to multiple parameters of the algorithm. Furthermore, it can be used to calculate the statistical significance of results. Specifically, the probability of observing a binding site with at least $R$ tags is given by the sum of Poisson probabilities below [50].

$$
1-\sum_{k=0}^{R-1}\left(\frac{e^{-\lambda} \lambda^{k}}{k !}\right)
$$

Once the computation is complete, the results are displayed as a set of chromosome coordinates.

\subsection{Strengths and Limitations}

There are several strengths and limitations when utilizing the SISSRs algorithm for determining true binding sites from ChIP-Seq data. However, it is possible to tune the SISSRs algorithm in an application specific manner; namely by modifying the threshold values that govern sensitivity and specificity as discussed in section 3.1.. While it does require a certain amount of mastery over the software, tuning these parameters allows the user to fit the results to their purposes.

Overall, SISSRs boasts superior sensitivity and specificity. For example, Jothi's application of SISSRs on the well-known and widely studied transcription factors CTCT, NRSF, and STAT1 identified 26184, 5813, and 73956 binding sites, respectively. This represents a significant increase in the number of binding sites previously proposed to exist, with increases of $32 \%, 299 \%$, and $78 \%$ for CTCT, NRSF and STAT1, respectively.

Furthermore, an overwhelming majority of the identified sites overlapped with previously identified sites, this indicating that the SISSRs algorithm predicts binding sites accurately [51].

Furthermore, the SISSRs algorithm possesses a high resolution, meaning the sites identified are very close to the actual binding site - within a few tens of base pairs. In comparison, past methods of analysis determined binding "regions" instead of sites, which were hundreds to a few thousand base pairs in length. As a result, SISSRs have allowed scientists to go from a general image containing 
Volume 8 Issue 9 September 2020

clutters of multiple binding sites to pinpointing sites very accurately.

The number of tunable parameters in the SISSRs algorithm makes this technology very flexible for a broad user base. For example, low-quality ChIP-Seq data may contain numerous false positives, sources of background noise, non-specificity, and errors. By changing the size of the scanning window used by the algorithm, the user can reduce the impact of these flaws at the cost of lower resolution (wider scanning window). In addition, the program is compatible with control data, thereby subtracting sequencing errors and background noise to some degree.

However, the software is not without its limitations. It is not immune to errors and false positives, and is highly impacted by the quality of ChIP-Seq data it receives. ChIP-Seq data that are obtained using antibodies of poor quality (non-specific) or other poorly performing methods inevitably degrade the performance of the algorithm. In these cases, the blame should be placed on the limitations of ChIP technology, not SISSRs. Overall, the ability for SISSRs to mitigate the negative effects of poor quality data, while providing results of unprecedented accuracy and sensitivity, certainly makes it a promising technology.

\section{NGS}

Sequencing is a component of ChIP-Seq that is worth discussing. As mentioned, ChIP-Seq technology takes advantage of the widely available NGS to sequence the fragments that have been precipitated. NGS has not only massively improved and completed ChIP, but it has also revolutionized biological and clinical fields of science. With the addition of throughput power and the large removal of sequencing bias, NGS completed its namesake job: taking biology to its next generation.

\subsection{NGS Workflow}

NGS has a three-step execution: library preparation, amplification, and sequencing. In general, the DNA is fragmented and ligated to adapters during library preparation, which facilitates amplification. PCR takes place during amplification, and the resulting fragments are sequenced in parallel during the last step. Although there are many variations across different methods and purposes of NGS, they generally follow the same principle. Fragments of the DNA being sequenced are amplified on a flow cell, and they are sequenced massively in parallel through fluorescently labeled deoxyribonucleotide triphosphates (dNTP) that identify nucleotide bases.

\subsubsection{Library Preparation}

Library preparation refers to creating DNA fragments and preparing said fragments for subsequent steps of the NGS workflow. During ChIP-Seq, the former does not need to take place as the DNA is already fragmented - either through nuclease digestion or sonication - during the ChIP process. Instead, the pieces are prepared for PCR using adapters. Adapters allow library hybridization to flow cells and provide priming sites, which will be discussed later. When sequencing multiple libraries at once, adapters can be "barcoded" to distinguish between different libraries [52].

Adapters are short, double stranded oligonucleotides that are chemically synthesized and can be ligated to fragments. For NGS, they are engineered to have a "blunt" end, which is composed of 3'-OH and 5'-P ends that attach to the 5'-P and 3' $\mathrm{OH}$ ends, respectively, at the blunt ends of a DNA fragment, and a "sticky" end, which is created by an overhang of the 5'-P end. For the adapter and fragment to be ligated, the 3' end of the DNA fragment is adenylated, or A-tailed [53].

During the ligation reaction itself, there is a need to limit the number of adapters used because adapters can form dimers among themselves. When dimers are formed between adapters, they are often removed with magnetic beads.

During NGS, the sticky end of the adapter is usually dephosphorylated to a 5'-OH end instead of a 5'-P end. This prevents a dimerization between two fragments during ligation reactions, because the modified 5'-OH terminus of one adapter can no longer bind to the 3'-OH terminus of another. Otherwise, the formation of said dimers can obscure the workflow because it can be difficult to separate them [54]. 
Volume 8 Issue 9 September 2020

Once the fragments have been ligated at both ends, the library can be evaluated quantitatively and qualitatively before it is be sequenced. Often, the quantitative evaluation is done through Quantitative PCR (qPCR) and qualitative evaluation is done through Bioanalyzer prior to amplification. qPCR selectively amplifies adapter-ligated fragments to quantify the fragments that can be sequenced from the library. It is a very sensitive yet time-consuming procedure. Bioanalyzer analyzes the size distribution of the completed library, verifying that the fragment sizes before and after the library preparation are consistent [55].

\subsubsection{Amplification}

Several types of PCRs can be used to amplify the fragments, and the adapter provides the primer binding site for the reaction. Adapters are critical to the success of the PCR because certain characteristics, such as the Guanine-Cytosine (G-C) content, may heavily affect the bias of the results [56]. Among the varieties of PCR, Bridge PCR (bPCR) is used in the most common NGS platform, Illumina.

Once the library preparation is complete, the fragments are loaded onto a flow cell - a glass slide with distinct lanes. The flow cell is coated with a "lawn" of oligonucleotides that are complementary to the adapters ligated to the DNA fragments. The adapters are hybridized to the oligos on the surface of the flow cell, and the fragments are ready for amplification [57]. It is important that the spacing of fragments is regulated because when each fragment is amplified, they will create cultures/colonies that must be distinct from each other.

During bPCR, the unattached end of the singlestranded DNA fragment attaches to the flow cell, forming a "bridge." Subsequently, the reaction is executed, and elongation results in the formation of a double-stranded DNA. Denaturation separates a strand so that another cycle can be completed; repetitions of the cycle generates the desired colony. It is important to limit the cycle repetitions in order to prevent overcrowded clusters and to prevent clusters from interfering with each other [54]. While relatively simple, a problem with bPCR is that a fresh mixture of reagents, including polymerase, must be added at the start of each cycle [58].

Another less commonly used type of PCR is Emulsion PCR (emPCR). During emPCR, millions of microbeads are isolated in emulsion droplets, and a single stranded DNA anneals to the microbead. The polymerase amplifies the annealed DNA thereby creating an exact copy. Subsequently, the original strand is released and re-annealed to repeat the cycle, which occurs until enough copies have been formed [54]. It is a comparatively timeconsuming method because of the "emulsion breakup, template enrichment, and bead deposition" that must occur [58].

\subsubsection{Sequencing}

Finally, the fragments are sequenced in parallel, completing the process of recording raw genetic data. The most used platform, Illumina, utilizes reversible terminators in a sequence by synthesis approach; hence its name, Illumina SBS. It is considered the most efficient method that is currently in use.

In this method, the primer bound to the adapter is elongated by fluorescently labeled dNTPs, one by one. These dNTPs are separate molecules (A, T, G, C) and bind to their respective bases in a mechanism of natural competition. The fragments are exposed to dNTPs, and starting from the primer, they are added/attached in a downward direction. Since they serve as reversible terminators for polymerization, after the incorporation of a dNTP no more bases can be added until the fluorescent label is removed [59]. Thus, at each cycle, dNTP attaches to the strand, the cell is imaged, and the fluorophore branch is cleaved enzymatically, allowing for the continuation of subsequent cycles. dNTPs that have not been attached are washed away [54].

These cycles are executed in parallel for all clusters. At each cycle, a base is labeled through the signal intensity measurement from the fluorescent image of the flow cell; the result is a growing sequence for 
Volume 8 Issue 9 September 2020

each fragment. Since there are many copies of the same fragment in each cluster, the reads are very accurate, without any context-specific errors. Furthermore, since dNTPS are attached through natural competition, the bias usually associated with base incorporation is minimized.

Once the fragments have been fully sequenced, the reads can be aligned to a reference genome using bioinformatics software. This paves the way for comparative analysis between the reference and novel genome.

\subsection{Impact of NGS on Biological Science}

NGS was a significant step forward for all branches of biological science as it provides -omics level data at both DNA and RNA levels. With the ability to sequence entire genomes or transcriptomes, comes significant advantages to uncovering biological mechanisms. NGS has transformed what used to be either an abstract process of fishing through heritable and inheritable traits or extremely costly comprehensive analyses, to a rapid, mass generation of computable data that can be mined for potential leads.

The most notable example of the impact of NGS on the biological sciences is likely the Human Genome Project (HGP), which endeavored to map out the entire human genome. The first genome took 13 years and $\$ 1$ billion to sequence; now it takes one or two days and a thousand dollars. As a result, it hints at the possibility of personalized medicine in the near future, where patients are assigned treatments based on their genes, which may indicate susceptibility to certain diseases. Personalized medicine may also be used to prevent health problems before they even surface, such as cancers, where heritable genetic mutations play a major role in 10 percent of all cancers.

The potential impact of NGS on the medical field is in offering a more systematic, personalized approach to health care to the general public [60]. For scientists, NGS has already completely revolutionized the way that biomedical research is performed within only a few years.
There has been a dramatic proliferation of tools that enhance the data collection and interpretation of sequencing data [60]. Scientists have been developing new ways to use NGS and new methods to improve accuracy, accessibility, and cost. This is primarily achieved through the development of new platforms and algorithms. For example, some platforms are beginning to incorporate nanotechnology [61] or electron microscopy. Moreover, increasing numbers of software tools have been developed to analyze NGS data, such as the aforementioned SISSRs. These approaches are valuable for data interpretation, and they depend on the digital nature of NGS.

As a result, scientists are able to study biological phenomena that were previously too complex, such as variant detection, rare variation testing, identification of de novo mutations, rare mendelian disorders, and somatic variation detection [62]. Furthermore, NGS has allowed them to construct massive databases containing annotated genomes of multiple organisms, many of which are available to the public. This system of genomic annotations has risen in popularity with the establishment and expansion of sequence databases. Although they are controversially prone to errors, it is undeniable that annotations have invaluable potential as they are capable of providing quality data that can be integrated into genomic maps [63].

NGS has become prevalent in many biological sciences due to its versatility. The most popular areas of research are clinical virology, bacteriology, and interrogation of genetic mutations. The highthroughput sequencing allows scientists to quickly profile and analyze novel viruses [64], develop countermeasures against infectious diseases [65], and detect mutations that result in potential health issues, such as genetic diseases and cancers [66].

In summary, NGS prompts scientists to fully harness the technology of the digital age. NGS empowers scientists by massively increasing the amount of data that can be generated about a biological process. This has proven dramatically superior to older traditional methodologies which were often tedious, and more qualitative rather than 
Volume 8 Issue 9 September 2020

computable. Lastly, it has increased the availability of data and information to the general public, thus enabling a wider demographic to conduct research.

\section{METHOD MODIFICATIONS}

Without a doubt, ChIP-Seq has revolutionized the study and interrogation of protein-DNA interactions by combining a highly specific and sensitive, in vivo method (ChIP) with the ability to sequence DNA samples with minimal bias, massive throughput, and high accuracy without the need for context (NGS). Compared to its original counterpart, ChIP-on-chip, which uses microarray hybridization of DNA fragments [67], ChIP-Seq boasts a higher resolution, coverage, and costefficiency, in addition to a smaller amount of required material and bias [68]. With an access to a wider arsenal of genomic technology, ChIP-Seq clearly emerges as the superior in terms of performance.

However, ChIP-Seq is not without its flaws. ChIPSeq is very expensive - cost ranging from $\$ 1000$ to $\$ 2000$ for Illumina. While it is true that recent advances in NGS have contributed to lower sequencing costs, ChIP itself remains an expensive tool. Furthermore, ChIP has a very low availability. The materials required to execute ChIP are often not commercially available, making it more fit in the setting of labs. For example, immunoprecipitation requires highly specific antibodies, the quality of which are critical to the quality of the results, which can be a limitation when investigating proteins with insufficient information. Using the wrong antibodies can taint the specificity, a normally extolled characteristic of ChIP.

Although its resolution and accuracy are superior to that of ChIP-on-chip, it does not mean that ChIPseq possesses perfection in those categories. Because sonication itself is not a very specific process, fragments can be a few hundred bases longer than the length of the actual binding sites. Furthermore, there is no way to remove false positives that arise due to contamination during the ChIP component.
In addition, meaningful results from ChIP-Seq also requires the presence of control data, which not only complicates the process, but also exacerbates the problem of cost since multiple trials are required for each of its steps. As briefly mentioned in the description of ChIP protocol, steps such as sonication, PCR, and immunoprecipitation are prone to notable degrees of bias, unless they are optimized through control data. It may take multiple trials before the best conditions for each of the steps can be obtained. As demonstrated by the SISSRs Algorithm, having control data can also be critical to the accuracy of algorithms that are run on the raw data generated by ChIP-Seq.

Thus, ChIP-Seq is still a maturing technology, that has yet to become widely available for public use. Despite its high accuracy and coverage, its high costs and complexity hampers its popularity to some scientists. More importantly, ChIP-Seq can be further complemented by other technologies to further increase its target specificity and accuracy. To this end, there are various alternative methods to ChIP-Seq that are employed in the study of genetics. The following section provides insight into two other technologies that are similar in nature to ChIP-Seq: PLAC-Seq and ChIP-exo.

\subsection{PLAC-Seq}

Proximity Ligation-Assisted ChIP-Seq (PLAC-Seq) is a modification to ChIP-Seq that is utilized in studying long range chromatin interactions. Long range chromatin interactions are mostly centered around chromatin looping, which is essential to that activation of transcription as mentioned in the Transcription Factor section of this paper. Briefly restated, the interactions between separate enhancers and promoters that create chromatin looping are responsible for increasing the concentration of materials necessary for transcription; thus, transcription is activated.

Before the introduction of PLAC-Seq, a conventional method tailored for this purpose was Chromatin Interaction Analysis by Paired-End Tag Sequencing (ChIA-PET). While a relatively costefficient method that can produce high-resolution results, it requires hundreds of millions of cells to 
Volume 8 Issue 9 September 2020

produce results. This is likely because it shears the chromatin prior to proximity ligation. Instead, PLAC-Seq reverses the order by shearing the chromatin after proximity ligation, resulting in a superior efficiency and accuracy [69].

The PLAC-Seq workflow is quite similar to ChIPSeq with the addition of proximity ligation. Cells are first fixed with formaldehyde. This is followed by a biotin fill in (nucleotides tagged with biotin) and proximity ligation [70]. Subsequently, the nucleus is lysed, allowing for chromatin sonication and chromatin immunoprecipitation to occur. Finally, the precipitated fragments are reversecrosslinked are enriched and purified, ending with paired-end sequencing of the fragments.

Empirical data clearly demonstrate that PLAC-Seq is far superior to ChIA-PET. PLAC-Seq on RNA Polymerase II required 5 million cells compared to the 100 million required for ChIA-PET on Polymerase II. Under these conditions, the former sequenced 175 million total pairs, of which $89 \%$ were cis pairs and $67 \%$ were long-range cis pairs, within a time span of four days. On the other hand, the latter sequenced only 16 million total pairs, of which $52 \%$ were cis pairs and $8.6 \%$ were longrange cis pairs, within a time span of 6 days [71]. The performance of PLAC-Seq was drasticafly more rewarding than that of ChIA-PET, proving its efficiency in interrogation of long-range chromatin interactions.

As a result, ChIP-Seq can be applied to different means, and its specificity and accuracy is conserved when done right. PLAC-Seq accentuates one of the numerous ways in which this technology can be employed to advance studies in genetics.

\subsection{ChIP-exo}

ChIP-exo is a modified form of ChIP-Seq that serves the common purpose of mapping proteinDNA binding sites across the genome. ChIP-Seq is quite popular for its high resolution; ChIP-exo achieves an even higher resolution at almost a hundred-to-one ratio, boasting a near single nucleotide accuracy. ChIP-exo is intended to increase specificity and reduce background noise caused by non-specific genomic DNA.

ChIP-exo derives its name from chromatin immunoprecipitation and exonuclease digestion, and it is completed by NGS once the two have been completed. The specificity when using ChIP alone is inhibited by sonication due to its heterogeneity. In other words, sonication does not pinpoint the site of protein-DNA interactions; it fragments the DNA and by chance, general regions of such sites are captured. Using lambda exonuclease, this limitation can be eliminated.

Lambda exonuclease trims or digests unbound double-stranded DNA in a 5' to 3' direction. Thus, when it is used on a fragmented DNA that has been immunoprecipitated - without being reverse cross linked - it will digest the DNA from either end until it reaches the binding site [70]. Furthermore, when deployed on false positives due to contamination or false negatives, it can correct the mistakes, as the exonuclease digests all unbound portions of the DNA, leaving the bound regions intact. Through its mechanism, the exonuclease narrows down the binding site to an extremely high resolution, in addition to drastically reducing the background noise.

Application of ChIP-exo to DNA binding protein Reb1 of Schizosaccharomyces pombe (Fission Yeast) demonstrated that it could identify individual binding locations among protein clusters, which are mostly unattainable by ChIP-Seq or ChIP-on-chip. Considering that 60 percent of 1,776 identified peaks were primary locations or clusters, this is quite significant to the improvement of specificity. Furthermore, the false positive rate was less than $0.05 \%$ in the ChIP-exo dataset, demonstrating the high accuracy of this protocol [71].

ChIP-Seq is flawed because its specificity, while substantial, is not at its maximum potential, leading to ambiguity of binding sites. Furthermore, it is also subject to false-positives and background noise because there isn't a protocol to confirm that the obtained DNA fragments are truly bound to proteins. Through the addition of the lambda 
Volume 8 Issue 9 September 2020

exonuclease, these aspects are drastically improved in ChIP-exo.

\section{COMMON ANALYSIS TOOLS}

The final section explores the different tools that are applied to the obtained ChIP-Seq data for means of analysis. ChIP-Seq is an excellent technology for acquiring raw data, but it is rendered meaningless without proper data analysis. Proper steps in analysis includes aligning sequences and aggregating data for statistical and comparative analysis. The most popular tools currently in use are MultiQC and ELAND.

\subsection{MultiQC}

MultiQC is a bioinformatics tool that is popular for its ability to combine data from multiple samples into a single report. This facilitates comparison between data, providing insight into differences and similarities that could have easily been overlooked, had the data been separate. By compiling data across multiple samples in a single summary, it makes it easier for the user to scan through statistics of interest. Thus, it removes the need for the repetition of finding and compiling reports from single samples, which is not only time consuming but also subject to potential errors.

MultiQC generates an easily accessible HTML report that contains interactive plots. The user can manipulate features on the report: hiding or highlighting samples and resizing plots make the report easy to adjust based on user's interest. Although MultiQC itself is compatible with a wide variety of bioinformatics tools, a custom code makes it easy for users to control the functionality and inner workings of the program. Thus, it can be personalized for the specific individual needs of users.

With the ability to accommodate many samples at once, parse data with massive power ("thousands of samples within minutes"), and serve as a quality control for data, MultiQC is rising in popularity in the field of bioinformatics. It serves as the pipeline endpoint for the aggregation of multiple samples and is, therefore, best used in comparative studies of single cells and populations. Using this tool, it is possible to filter out bias and identify abnormal samples, which are derived from the convenience in placing multiple samples against one another.

This technology has revolutionized the platform that researchers use for data analysis; with a more convenient platform comes the reduction in time and work that is required for conventional methods of analysis [72].

\subsection{ELAND}

ELAND is a program developed by Illumina with the purpose of aligning short sequence reads to a reference genome in order to pinpoint genetic variations and is well known for its high sensitivity. More specifically, ELAND can guarantee full sensitivity for reads that contain a maximum of 2 mismatches with the reference. It is a fast and accurate software that is useful in combining short reads that were generated in parallel and detecting SNPs, which capture genetic variation in a population Jdentifying SNPs can be extremely useful in indexing causes for genetic diseases [73].

In order to do this, ELAND divides a read into four parts and creates six "seed templates" through a combination of two of the four parts. The templates are applied on the reference genome to index it, and then the reference genome is scanned base-by-base to find hits/mismatches. As a result, ELAND efficiently aligns the reads to the reference genome and identifies all reads with 2 hits [74]. ELAND's sequence read alignment and identification of mismatches in said aligned sequences is crucial to pinpointing mutations and structural rearrangements in samples [75].

Despite its efficiency and accuracy, ELAND has its limitations because it is incapable of supporting reads longer than 32bp at the same power and sensitivity. If the first 32 bases of an aligned read are not unique, ELAND has an increased probability of missing the alignment, as it mostly depends on hits or mismatches. Novel algorithms and ELAND itself continue to adapt in order to overcome such limitations. 
Volume 8 Issue 9 September 2020

\section{CONCLUSION}

The investigation of protein-DNA binding sites is one of the crucial components to understanding the biology of organisms. Protein-DNA interactions govern many biological processes at a cellular level, and the visualization and comprehension of those processes aid scientists in identifying connections between seemingly separate concepts or phenomena. ChIP-Seq is one of the best tools tailored for this purpose, as it inherently assays interactions between proteins and DNA.

While ChIP-Seq has flaws, alternatives such as ChIP-exo or PLAC-seq prove weaker in the areas where ChIP-Seq excels. As a result, it would be beneficial to consider the cost and benefits of each method, or to run multiple trials to empirically determine which methodology meets the intended application.

Another reason for the popularity of ChIP-Seq is its versatility. The NGS component provides the researcher with data that are compatible with many different software packages. One can refine such data with algorithms such as SISSRs peakfinder, or analyze and interpret the data with programs such as MultiQC and ELAND.

Bioinformatics is a relatively new field of study, but advanced tools like ChIP-Seq open up an infinitude of possibilities. Scientists are just beginning to uncover the true potential of incorporating such technologies into the biological sciences.

\section{REFERENCES}

[1]. Das, P., Ramachandran, K., vanWert, J., \& Singal, R. (2018). Chromatin immunoprecipitation assay.

BioTechniques, 37(6). doi:https://doi.org/10.2144/04376RV01

[2]. Tretyakova, N., Groehler, A., \& Shaofei, J. (2015). DNA-Protein Cross-links: Formation, Structural identities, and Biological Outcomes. Acc Chem Res, 48(6), 1631-1644. doi:https://doi.org/10.1021/acs.accounts.5b 00056
[3]. Milne, T., Zhao, K., \& Hess, J. (2009).

Chromatin Immunoprecipitation (ChIP) for

Analysis of Histone Modifications and

Chromatin-Associated Proteins. Methods

Mol Biol(538), 409-423. From

10.1007/978-1-59745-418-6_21

[4]. Mukhopadhyay, A., Deplancke, B., Wallhou, A., \& Tissenbaum, H. (2008). Chromatin immunoprecipitation (ChIP) coupled to detection by quantitative real-time PCR to study transcription factor binding to DNA in Caenorhabditis elegans. Nat Protoc, 3(4), 698-709. doi:10.1038/nprot.2008.38

[5]. Gade, P., \& Kalvakolanu, D. (2012). Chromatin Immunoprecipitation Assay as a Tool for Analyzing Transcription Factor Activity. Methods Mol Biol, 809, 85-104. From 10.1007/978-1-61779-376-9_6

[6]. Giner-Lamia, J., Hernandez-Prieto, M., \& Futschik, M. (2018). ChIP-seq Experiment and Data Analysis in the Cyanobacterium Synechocystis sp. Pcc 6803. BioProtocol, 8(12). doi:10.21769/BioProtoc.2895

[7]. Behjati, S. T. (2013). What is next generation sequencing? Arch Dis Child Educ Pract Ed, 98(6), 236-238. doi:10.1136/archdischild-2013-304340

[8]. Meyer, C., \& Liu, X. (2014). Identifying and Mitigating Bias in Next-Generation Sequencing Methods for Chromatin Biology. Nat Rev Genet, 15(11), 709-721. doi:10.1038/nrg3788

[9]. Zirong, L., \& al., e. (2003). A global transcriptional reole for c-Myc in Burkitt's lymphoma cells. Proc Natl Acad Sci USA, 100(14), 8164-8169. doi:10.1073/pnas.1332764100

[10]. Travers, A. A. (1993). DNA-protein interactions. London: Springer.

[11]. Brivanlou, A., \& Darnell, J. (2002). Signal Transduction and the Control of Gene Expression. Science, 295(5556), 813-818. doi:10.1126/science.1066355

[12]. Phillips, T. (2019). Transcription Factors. Retrieved February 9, 2020 from ThoughtCo: https://www.thoughtco.com/what-aretranscription-factors-375675 
Volume 8 Issue 9 September 2020

[13]. Eberwine, J. (1999). Regulation of Transcription by Transcription Factors. In Basic Neurochemistry: Molecular, Cellular and Medical Aspects. Philadelphia: Lippincott-Raven.

[14]. Latchman, D. (1997). Transcription factors: An overview. The International Journal of Biochemistry \& Cell Bioloy, 29(12), 13051312. doi:https://doi.org/10.1016/S13572725(97)00085-X

[15]. Warnmark, A., Treuter, E., Wright, A., \& Gustafsson, J. (2003). Activation Functions 1 and 2 of Nuclear Receptors: Molecular Strategies for Transcriptional Activation. Molecular Endocrinology, 17(10), 1901. doi:https://doi.org/10.1210/me.2002-0384

[16]. Hoppe, T., Rape, M., \& Jentsch, S. (2001). Membrane-bound transcription factors: regulated release by RIP or RUP. Current Opinion in Cell Biology, 13(3), 344-348. doi:https://doi.org/10.1016/S09550674(00)00218-0

[17]. Lomvardas, S., Barnea, G., Pisapia, D., Mendelsohn, M., Kirkland, J., \& Axel, R. (2006). Intrachromosomal Interactions and Olfactory Receptor Choice. Cell, 126(2), P403-413. doi:https://doi.org/10.1016/j.cell.2006.06.0 35

[18]. Pennacchio, L., Bickmore, W., Dean, A., Nobrega, M., \& Bejerano, G. (2013). Enhancers: five essential questions. Nat Rev Genet, 14(4), 288-295. doi:10.1038/nrg3458

[19]. Cooper, S., Trinklein, N., Anton, E., Nguyen, L., \& Myers, R. (2006). Comprehensive analysis of transcriptional promoter structure and function in $1 \%$ of the human genome. Genome Res, 16(1), 1-10. doi:10.1101/gr.4222606

[20]. Dao, L., \& Spicuglia, S. (2018).

Transcriptional regulation by promoters with enhancer function. Transcription, 9(5), 307-314. doi: $10.1080 / 21541264.2018 .1486150$
[21]. Lee, T., \& Young, R. (2013). Transcriptional Regulation and Its Misregulation in Disease. Cell, 152(6), P1237-1251. doi:https://doi.org/10.1016/j.cell.2013.02.0 14

[22]. Griffiths, A. e. (2000). An Introduction to Genetic Analysis. New York: W.H. Freeman.

[23]. Nikolov, D., \& Burley, S. (1997). RNA polymerase II transcription initiation: A structural view. PNAS, 94(1), 15-22. doi:https://doi.org/10.1073/pnas.94.1.15

[24]. Zaman, Z., Ansari, A., Koh, S., Young, R., \& Ptashne, M. (2001). Interaction of a transcriptional repressor with the RNA polymerase II holoenzyme plays a crucial role in repression. PNAS, 98(5), 25502554.

doi:https://doi.org/10.1073/pnas.04161119 8

[25]. Sperling, S. (2007). Transcriptional regulation at a glance. BMC Bioinformatics, 8(6), S2. doj: 10.1186/1471-2105-8-S6-S2

[26]. Yeo, J., \& Ng, H. (2013). The transcriptional regulation of pluripotency. Cell Research, 23, 23-32. doi:https://doi.org/10.1038/cr.2012.172

[27]. Lambert, M., Jambon, S., Depauw, S., \& David-Cordonnier, M. (2018). Targeting Transcription Factors for Cancer Treatment. Molecules, 23(6), 1479. doi:10.3390/molecules 23061479

[28]. Bushweller, J. (2019). Targeting transcription factors in cancer - from undruggable to reality. Nature Reviews Cancer, 19, 611624. doi:https://doi.org/10.1038/s41568019-0196-7

[29]. Boheler, K. (2009). Stem Cell Pluripotency: A Cellular Trait that Depends on Transcription Factors, Chromatin State and a Checkpoint Deficient Cell Cycle. J Cell Physiol, 221(1), 10-17. From 10.1002/jcp.21866

[30]. Rathnam, C., Cheung, S., \& Lee, K. (2017). Advanced Gene Manipulation Methods for Stem Cell Theranostics. Theranostics, 17(1), 2775-2793. doi:10.7150/thno.19443 
Volume 8 Issue 9 September 2020

[31]. Luger K, Mäder A, Richmond R, Sargent D, Richmond T (1997). "Crystal structure of the nucleosome core particle at $2.8 \mathrm{~A}$ resolution". Nature. 389 (6648): 251-60.

[32]. Kubicek, S., \& al., e. (2006). The Role of Histone Modifications in Epigenetic Transitions During Normal and Perturbed Development. In S. Berger, O. Nakanishi, \& B. Haendler, The Histone Code and Beyond. Berlin, Heidelberg: Springer.

[33]. Alhamwe, B., \& al., e. (2018). Histone modifications and their role in epigenetics of atopy and allergic diseases. Allergy Asthma Clin Immunol, 14(39). doi:10.1186/s13223-018-0259-4

[34]. Swygert, S., \& Peterson, C. (2014). Chromatin dynamics: interplay between remodeling enzymes and histone modifications. Biochim Biophys Acta, 1839(8), 728-36. doi:10.1016/j.bbagrm.2014.02.013

[35]. Rossetto, D., Avvakumov, N., \& Jacques, C. (2012). Histone phosphorylation. Epigenetics, 7(10), 1098-1108. doi:10.4161/epi.21975

[36]. Edmondson, D., \& al., e. (2002). Site-specific Loss of Acetylation upon Phosphorylation of Histone H3. The Journal of Biological Chemistry, 277. From 10.1074/jbc.M200651200

[37]. Cao, J., \& Yan, Q. (2012). Histone Ubiquitination and Deubiquitination in Transcription, DNA Damage Response, and Cancer. Front Oncol, 2(26). doi:10.3389/fonc.2012.00026

[38]. Weake, V., \& Workman, J. (2008). Histone Ubiquitnation: Triggering Gene Activity. Molecular Cell, 29(6), P653-663. doi:https://doi.org/10.1016/j.molcel.2008.0 2.014

[39]. Zhang, X., \& al., e. (2017). H3 ubiquitination by NEDD4 regulates $\mathrm{H} 3$ acetylation and tumorigenesis. Nat Commun, 8(14799). doi:10.1038/ncomms 14799

[40]. Ohguchi, H., Hideshima, T., \& Anderson, K. (2018). The biological significance of histone modifiers in multiple myeloma: clinical applications. Blood Cancer
Journal, 8(9), 83. doi:10.1038/s41408018-0119-y

[41]. Keats, J., \& al., e. (2005). Overexpression of transcripts originating from the MMSET locus characterizes all $\mathrm{t}(4 ; 14)(\mathrm{p} 16 ; \mathrm{q} 32)-$ positive multiple myeloma patients. Bklood, 105(10), 4060-4069. doi:10.1182/blood-2004-09-3704

[42]. Pei, H., \& al., e. (2011). MMSET regulates histone H4K20 methylation and 53BP1 accumulation at DNA damage sites. Nature, 470(7332), 124-128. doi:10.1038/nature09658

[43]. Rutherford, E., \& Lowery, L. (2016). Exploring the developmental mechanisms underlying Wolf-Hirschhorn Syndrome: Evidence for defects in neural crest cell migration. Dev Biol, 420(1), 1-10. doi:10.1016/j.ydbio.2016.10.012

[44]. Weinhold, B. (2006). Epigenetics: the Science of Change. Environ Health Perspec, 114(3), A160-A167. doi:10.1289/ehp.114a 160

[45]. O'Geen, H., Echipare, L., \& Farnham, P. (2011). Using ChIP-Seq technology to generate high-resolution profiles of histone modifications. Methods Mol Biol, 791, 265-286. From 10.1007/978-1-61779-3165_20

[46]. Du, Z., \& al., e. (2013). Genome-Wide Analysis of Histone Modifications: H3K4me2, H3K4me3, H3K9ac, and H3K27ac in Oryza sativa L. Japonica. Molecular Plant, 6(5), 1463-1472.

[47]. Karlic, R., Chung, H., Lasserre, J., Vlahovicek, K., \& Vingron, M. (2010). Histone modification levels are predictive for gene expression. PNAS, 107(7), 29262931.

doi:https://doi.org/10.1073/pnas.09093441 07

[48]. Kelley, D., \& al., e. (2017). Integrated analysis of whole-genome ChIP-Seq and RNA-Seq data of primary head and neck tumor samples associates HPV integration sites with open chromatin marks. Cancer Research. Retrieved February 9, 2020 from https://cancerres.aacrjournals.org/content/c 
Volume 8 Issue 9 September 2020

anres/early/2017/09/23/0008-5472.CAN17-0833.full.pdf

[49]. Sadri-Vakili, G., \& Cha, J. (2006).

Mechanisms of disease: Histone

modifications in Huntington's disease. Nat Clin Pract Neurol, 2(6), 330-8.

[50]. Narlikar, L., \& Jothi, R. (2012). ChIP-Seq Data Analysis: Identification of ProteinDNA Binding Sites with SISSRs PeakFinder. Methods MOl Biol, 802, 305-322. doi:10.1007/978-1-61779-400-1_20

[51]. Jothi, R., Cuddapah, S., Barski, A., Cui, K., \& Zhao, K. (2008). Genome-wide identification of in vivo-protein- DNA binding sites from ChIP-Seq data. Nucleic Acids Res.,, 36(16), 5221-5231. Retrieved February 9, 2020 from https://www.ncbi.nlm.nih.gov/pmc/articles /PMC2532738/

[52]. Next Generation Sequencing (NGS) An Introduction. (n.d.). Retrieved February 9, 2020 from ABM:

https://www.abmgood.com/marketing/kno wledge_base/next_generation_sequencing _ introduction.php

[53]. Head, S., \& al, e. (2014). Library construction for next-generation sequencing: Overviews and challenges. Biotechniques, 56(2), 61 passim. doi:10.2144/000114133

[54]. Next generation sequencing. (n.d.). Retrieved February 9, 2020 from ATDBio: https://www.atdbio.com/content/58/Nextgeneration-sequencing

[55]. Next Generation Sequencing (NGS)

Experimental Design. (n.d.). Retrieved February 9, 2020 from ABM: https://www.abmgood.com/marketing/kno wledge_base/next_generation_sequencing_ experimental_design.php

[56]. Lorenz, T. (2012). Polymerase Chain Reaction: Basic Protocol Plus Troubleshooting and Optimization Strategies. Journal of Visualized Experiments, 63(3998). doi:10.3791/3998

[57]. An introduction to Next-Generation

Sequencing Technology. (n.d.). Retrieved February 9, 2020 from Illumina: https://www.illumina.com/documents/prod ucts/illumina_sequencing_introduction.pdf

[58]. Ma, Z., \& al, 3. (2013). Isothermal amplification method for next-generation sequencing. Proc Natl Acad Sci USA, 110(35), 14320-14232. doi:10.1073/pnas.1311334110

[59]. Illumina Sequencing Technology. (n.d.). Retrieved February 9, 2020 from Illumina: https://www.illumina.com/documents/prod ucts/techspotlights/techspotlight_sequencin g.pdf

[60]. Zhang, J., Chiodini, R., Badr, A., \& Zhang, G. (2011). The impact of next-generation sequencing on genomics. J Genet Genomics, 38(3), 95-109. doi:10.1016/j.jgg.2011.02.003

[61]. Iqbal, S. M., Akin, D., \& Bashir R. (2007). Solid-state nanopore channels with DNA selectivity. Nat Nanotechnol, 2(4), 2438. doi:10.1038/nnano.2007.78

[62]. Koboldt, D. C., Steinberg, K. M., Larson D. E., Wilson, R. K., \& Mardis, E. (2013). The Next-Generation Sequencing Revolution and Its Impact on Genomics. Cell, 155(1), 27-38. doi:10.1016/j.cell.2013.09.006

[63]. Koonin, E. V. \& Galperin, M. Y. (2003). Genome Annotation and Analysis. Sequence-Evolution- Function: Computational Approaches in Comparative Genomics. Boston: Kluwer Academic.

[64]. Barzon, L., Lavezzo, E., Militello, V., Toppo, S., \& Palù, G. (2011). Applications of Next-Generation Sequencing Technologies to Diagnostic Virology. Int J Mol Sci, 12(11). doi:10.3390/ijms12117861

[65]. Motro, Y., Moran-Gilad, J. (2017). Nextgeneration sequencing applications in clinical bacteriology. Biomol Detect Quantif, 14, 1-6. doi: 10.1016/j.bdq.2017.10.002

[66]. Lee, C., Chiu, Y., Wang, L., Kuo, Y., Chuang, E. Y., Lai, L., Tsai, M. (2013). Common applications of next-generation sequencing technologies in genomic 
Volume 8 Issue 9 September 2020

research. Translational Cancer Research, 2(1). doi:10.3978/j.issn.2218676X.2013.02.09

[67]. Fullwood, M., \& al, e. (2009). An oestrogenreceptor-alpha-bound human chromatin interactome. Nature, 462(7269), 58-64. doi:10.1038/nature08497

[68]. Gustafsdottir, S., \& al, e. (2005). Proximity ligation assays for sensitive and specific protein analyses. Analytical Biochemistry, 345(1), 2-9. doi: https://doi.org/10.1016/j.ab.2005.01.018

[69]. Fang, R., Yu, M., Li, G., Chee, S., Liu, T., Schmitt, A., \& Ren, B. (2016). Mapping of long-range chromatin interactions by proximity ligation-assisted ChIP-seq. Cell Research, 26, 1345-1348. doi:https://doi.org/10.1038/cr.2016.137

[70]. Rhee, H., \& Pugh, B. (2013). ChIP-exo: A Method to Identify Genomic Location of DNA-binding Proteins at Near Single Nucleotide Accuracy. Curr Protoc Mol Biol. doi:10.1002/0471142727.mb2124s100

[71]. Rhee, S., \& Pugh, B. (2011). Comprehensive Genome-wide Protein-DNA Interactions Detected at Single Nucleotide Resolution. Cell, 147(6), 1408-1419. doi:10.1016/j.cell.2011.11.013
[72]. Ewels, P., Magnusson, M., Lundin, S., \& Kaller, M. (2016). MultiQC: summarize analysis result for multiple tools and samples in a single report. Bioinformatics, 32(19), 3047-3048. doi:10.1093/bioinformatics/btw354

[73]. Ingarm, V. (1956). A Specific Chemical Difference Between the Globins of Normal Human and Sickle Cell Anaemia Haemoglobin. Nature, 178(4537), 792-4. doi:10.1038/178792a0

[74]. attractivechaos. (2008). The Eland Short Read Aligner. Retrieved February 9, 2020 from Attractive Chaos: https://attractivechaos.wordpress.com/2008 /08/24/eland-short-read-aligner/

[75]. Genomic Sequencing. (n.d.). Retrieved February 9, 2020 from Illumina: https://www.illumina.com/documents/prod ucts/datasheets/datasheet_genomic_sequen ce.pdf 